\title{
Proceedings of the Society of British Neurological Surgeons Meeting in Liverpool, 27-28 October 1983
}

\section{STABILISATION OF THE SPINE IN METASTATIC MALIGNANCY \\ John Miles, AJ Banks, EH Dervin (Liver- pool)}

Spinal metastatic cancer is common and the authors therefore had reviewed the problems in its neurosurgical management. They considered that the results of treatment were depressing. Rarely did operations disclose a lesion such as a myeloma or a prostatic tumour, which could be expected to show worthwhile improvement. Both neurological deficits and pain could be due either to tumour compression or to skeletal instability. The customary treatment by laminectomy and evacuation of tumour might relieve tumour compression but at the same time could only increase instability. An operation was reported to stabilise the spine by means of a steel rod which was screwed into the posterior arch of adjacent vertebrae at the time of the exploratory laminectomy.' The technique had been applied to 20 patients with metastatic cancer and 3 with primary malignancy of their spine. It had enabled immediate mobilisation with freedom from pain and a prompt return to active life. Subsequent radiotherapy for the primary disease had not been precluded. The technique was not difficult but needed a surgeon of greater experience and expertise than was customarily possessed by those usually called on to perform urgent operations on patients with spinal malignancy. The authors had also used the technique in benign spinal lesions in two patients and had applied it to the anterior surface of the cervical spine in three cases; their findings emphasised the versatility of the technique and its practical value.

\section{Reference \\ ' Banks AJ, Dervin E. Engineering in Medicine 1980;9:81-83.}

ELECTROMECHANICAL COUPLING IN CEREBRAL VASCULAR SMOOTH MUSCLE IN RESPONSE TO SEROTONIN

A Waters (Cambridge)

This study was undertaken to define some of the basic cellular mechanisms of the action of serotonin on cerebral vascular smooth muscle. The author had used extremely sophisticated techniques to measure force development, intracellular membrane potential $\left(E_{m}\right)$, input resistance $R_{\text {in }}$ ) and $E_{m}$ versus log external potassium concentration curves. Isolated segments of cat basilar artery (approx. $3 \mathrm{~mm}$ in length) were used. The lumen of each segment was threaded with two lengths of 32 micron diameter tungsten wire. These wires were then stretched over the open jaws of two stainless steel rings. One ring was secured and the other connected to a very sensitive load cell (Kulite). Measurements of $E_{m}$ had employed glass microelectrodes filled with $3 \mathrm{M}$ potassium chloride, with tip resistance of $50-80$ Mohms and tip potentials less than $7 \mathrm{mV}$. All impalements were made from the adventitial surface. Electrical and mechanical events were recorded simultaneously in several experiments. Serotonin caused a dose dependent depolarization and contraction beginning at $3 \times 10-9 \mathrm{M}$. The correlation coefficient relating the degree of force development with the change in membrane potential was 0.98. Excess potassium depolarised the smooth muscle cells, and plots of the relationship between $E_{m}$ and external potassium concentration in the range $10-100 \mathrm{mM}$ showed a slope of $54 \mathrm{mV} /$ decade. Depolarisation of the membrane by excess $\mathrm{K}^{+}$resulted in the mechanical response to serotonin becoming significantly reduced; this indicated voltage inactivation of serotonin induced contraction. Steady state current/voltage curves demonstrated a reduction of input resistance; the author suggested that this showed that the depolarisation caused by serotonin was not due to a reduction in potassium conductance. Some arterial segments were chemically denervated with 6-hydroxy-dopamine to determine if part of the action of serotonin was mediated by norepinephrine release from adrenergic nerves. Destruction of adrenergic nerves did not alter the response to serotonin. $\mathrm{Mr}$ Waters concluded that his findings demonstrated that serotonin contracted the basilar artery through mechanisms that involved depolarisation of smooth muscle cells and that factors that influenced the level of $E_{m}$ affected markedly the contractile response to serotonin.

ROLE OF VASCULAR ENDOTHELIUM IN MAINTENANCE OF HUMAN CEREBRAL ARTERIAL TONE

RH Lye, YO Amure, ET Walley (Manchester)

It has been shown that several vasoactive substances contract smooth muscle in human cerebral arteries (for example, 5-hydroxytryptamine, prostaglandins, noradrenaline) whilst other agents cause relaxation (for example, prostacyclin, bradykinin, acetylcholine). Experiments on arteries of other species have demonstrated that the relaxant effect of bradykinin (BK) and acetylcholine (A Ch) depends on the presence of endothelial cells, and this stimulated the authors to investigate the effect of BKB and A Ch on human basilar artery preparations in the presence and absence of an intact endothelium. Necropsy human basilar artery (HBA) (obtained within 24 hours of death from patients dying from causes other than cerebrovascular disease) were cut into rings and mounted in tissue baths containing Krebs-Henseleit bubbled with $95 \% / \mathrm{O}_{2} / 5 \%$ $\mathrm{CO}_{2}$. De-endothelialised ring segments from the same arterial specimen were prepared by gently rubbing the endothelial surface to remove endothelial cells and these were then mounted in the same manner. After equilibration under $1 \mathrm{gm}$ resting tension, dose response curves to $\mathrm{BK}$ $\left(10^{-10}-10^{-6} \mathrm{M}\right)$ and $\mathrm{A} C \mathrm{Ch}\left(10^{-10}-10^{-4} \mathrm{M}\right)$ were obtained. Both $\mathrm{BK}$ and $\mathrm{ACh}$ produced dose-dependent relaxation in HBA preparations with intact endethelium. These relaxations were attenuated by BW775C $\left(10^{-5}-10^{-4} \mathrm{M}\right)$, a lipoxygenase/cyclo-oxygenase inhibitor. In de-endothelialised preparations the BK induced only weak relaxation whereas $A$ $\mathrm{Ch}$ produced contraction. The results indicated that the endothelial cells of HBA preparations produced a relaxing factor in 
a dose-dependent manner when challenged with BK or A Ch. Because indomethacin (a cyclo-oxygenase inhibitor) failed to block the relaxant effect of BK or A Ch on intact arterial preparations, the relaxing factor was probably a leukotriene or an hydroxyperoxy fatty acid metabolite. The authors concluded that the endothelial lining of cerebral arteries might have a modulating effect on the vascular tone maintained by the smooth muscle component of these vessels, and, furthermore, suggested that endothelial damage following subarachnoid haemorrhage might result in the imbalance in vaso motor tone and contribute to "spasm".

STEREOTAXIC CT INTEGRATION FOR BIOPSY AND INTERSTITIAL IRRADIATION OF CEREBRAL TUMOURS

F Afshar (London)

Stereotaxic CT guided biopsy is now an accepted neurosurgical procedure, but most methods require a general purpose scanner with a wide aperture or an expensive, purpose built, stereotaxic frame. The author had developed a method for steriotaxic CT biopsy of brain tumours using data from a single EMI 1010 CT scan section, transcribed to a standard Bennett Stereotaxic apparatus and reported its features and accuracy. He had designed a head frame and baseplate for use with the Bennett Stereotaxic frame and the CT 1010 scanner; after simple modification it could be used with any scanner. In the development of the system he had used phantoms designed specifically to identify the centre point of the stereotaxic frame and the scanner aperture, relative to the CT image. Precise target location was calculated using the statistic function software that was available for the CT 1010 scanner. This gave the $\mathrm{x}$ and $\mathrm{y}$ co-ordinates the axial plane. The distance of the lesion, at right angles to the base plate ( $\mathrm{z}$ co-ordinate) was calculated using an LED digital counter which recorded table top movement. This ensured reproducible pre- and postoperative scan slices. The development of specific screws and brackets made from polypropylene and tufnel bonded in resin, had enabled the production of scans that were virtually totally free from artefact distortion. The scan co-ordinates were converted (using a specific computer program) to the Bennett frame, which was attached to the baseplate. Biopsies were taken with a cannula that had a $1 \mathrm{~mm}$ internal diameter and which was fitted to a semi-circular arc attached to the stereotaxic frame. After biopsy a barium-impregnated spheroid was inserted and a repeat CT scan was undertaken to check the accuracy of the coordinates. Mr Afshar had performed a biopsy on fifteen patients and had obtained a positive result in each case. The accuracy was within 1-2 pixels in all cases and there was no morbidity or mortality. In discussion the value of stereotaxic biopsy was emphasised and the comparibility of the technique with other $\mathrm{CT}$ scanners was noted. In addition to providing material for neurological diagnosis, small, deep tumours could be treated by interstitial irradiation.

EXPLORATION OF THE TRIGEMINAL NERVE IN THE POSTERIOR FOSSA FOR TRIGEMINAL NEURALGIA

\section{TRK Varma, AK Frazer (Cardiff)}

The aetiology and best treatment of trigeminal neuralgia are uncertain. The authors reviewed twenty patients who had trigeminal neuralgia and who were treated by microsurgical operation on the trigeminal nerve in the posterior fossa; their aims were to determine how often there was evidence that local vascular lesions had caused the symptoms and how this correlated with the result of operation. They had found that it was difficult to judge if there was compression, but had noted "vascular contact" with the nerve in thirteen of the twenty patients. This contact was arterial in six patients, venous in four, and a combination in three others. When arterial contact was present it was found close to the pons whereas venous contact was found more laterally. In two patients an artery had passed between the fibres of the trigeminal nerve. In seven patients vascular contact was not found; these included two of three patients with multiple sclerosis. When a vascular contact was found the vessel was freed. Irrespective of whether vascular contact was present or not, the trigeminal nerve had been wrapped in muscle in each patient. Each of the patients with idiopathic trigeminal neuralgia had relief of pain, irrespective of the presence of vascular contact. None of these patients had experienced a recurrence during the follow-up period of 4-30 months. By contrast, two of the three patients with multiple sclerosis had not been relieved of their pain. In the third patient there had not been a vascular contact but the trigeminal nerve had been sectioned and this was followed by relief of pain. The authors' observations led to the conclusion that, although vascular contact was present in the majority of patients with idiopathic trigeminal neuralgia, muscle wrapping relieved pain irrespective of its presence or absence. While their findings supported the benefits of operation, there should be caution in making inferences between this and the pathogenesis of the pain. A useful practical point was that patients with multiple sclerosis did not benefit from "decompression" but did gain relief from root section.

A MULTI-CENTRE DOUBLE BLIND CONTROL LED TRIAL OF TRANEXAMIC ACID IN SUBARACHNOID HAEMORRHAGE

KW Lindsay, R Vermeulen, G Murray, F Cheah, A Hijdra, JP Muizelaar, M Schannong, GM Teasdale, H Van Creval, J Van Gijn (London, Glasgow, Rotterdam and Amsterdam)

Despite numerous trials of treatment with antifibrinolytic therapy of patients with subarchnoid haemorrhage, the value of this treatment remains uncertain. The authors considered that this was in part because the difficulty in the diagnosis of rebleeding had cast doubt on trials exposed to observer bias and also that trials showing "no significant reduction in re-bleeding rate" were inconclusive because all had an unacceptably high possibility of failing to show a significant difference. The reason for this was that each contained too few patients (false negative or Type II error Clinicians in the four centres had collabo rated in a randomised double blind study using a "pragmatic" approach, in order te्ष् determine the overall benefit of tranexamic acid in the management of subarachnoid haemorrhage. They had studied sufficient patients to ensure that there was little risk of the Type II error. Four hundred and seventy-four patients had been studied and each patient had received either tranexamic acid (241 patients) or placebo (238 patients). The important prognostic factors were well matched in each group but there was an imbalance in the numbers of patients who had a history of hypertension and also in the proportion who had an aneuyrsm demonstrated by angiography. When outcome was assessed three months after bleeding, there was no difference between the treatment group (35\% mortality) and the control group ( $37 \%$ mortality). Nevertheless, a striking finding was that the re-bleeding had been significantly less frequent in the group treated with tranexamic acid, but that also the incidence of cerebral infarction was significantly greater than in the controls. The analysis was adjusted to take account of the differences in the incidence of hypertension and of angiographic evidence of an aneurysm but this did not 
alter the findings. The authors concluded that they had demonstrated conclusively that, although tranexamic acid significantly reduced bleeding, because of its associated complications it did not confer overall benefits in the management of patients suffering from a subarachnoid haemorrhage.

THE PROGNOSTIC SIGNIFICANCE OF THE PRE-OPERATIVE CSF BICARBONATE LEVEL IN PATIENTS WITH RUPTURED INTRACRANIAL ANEURYM

J Jakubowski, R Blaszcyk, AA Jefferson, DMC Forster (Sheffield)

The mortality and morbidity of patients who undergo an operation to occlude an intracranial arterial aneurysm depends largely on the capacity of the cerebral circulatory reserve. The authors considered that this might be related to the size and distribution of ischaemic area and that, although these might be clinically silent initially, their presence might be reflected in the CSF base balance. They therefore had studied the relationship between CSF bicarbonate concentration, the demonstration of blood on the pre-operative CT scan and the incidence of post-operative neurological complications and had further related these to outcome after operation. Fifty-four patients suffering from a ruptured cerebral aneurysm had undergone operation; four were excluded from analysis because other factors had influenced the outcome. CSF samples were obtained through a lumbar catheter and were taken before operation, immediately after induction of anaesthesia, at the end of operation and also 8 and 24 hours later. The acid base balance was measured by gasometric methods and only patients with macroscopically clear CSF had been studied. Twenty-two patients had a normal CSF bicarbonate concentration $(22 \cdot 7 \pm 2 \cdot 8$ $\mathrm{mmol} / \mathrm{l})$ and had faired relatively well. Twenty-three per cent of these had a post-operative complication and in $68 \%$ the outcome 6-8 weeks after operation was judged to be either excellent or good. By contrast, 28 patients who had a low CSF bicarbonate concentration $(17.2 \pm 1.4$ $\mathrm{mmol} / \mathrm{l})$ had a high rate of post-operative neurological complications ( $82 \%$ ) and only $25 \%$ made an excellent or good recovery. The CT scan showed blood in the cisterns in similar proportions of the patients with either a normal or a low CSF bicarbonate concentration, but the incidence of postoperative neurological complications was particularly high $(91 \%)$ in patients with both a positive CT scan and a low CSF bicarbonate. Seven other patients, who died without surgery being performed, had a low CSF bicarbonate and evidence of blood on their CT scan. It was suggested that the significance and time course of changes in CSF biochemistry after subarachnoid haemorrhage should be established by further studies.

RUPTURED INTRACRANIAL ANEURYSMS: THE OVERALL IMPACT OF CURRENT METHODS OF TREATMENT AND THE EFFECT OF CASE SELECTION ON THE APPARENT OUTCOME

RS Maurice-Williams, H Marsh (London)

Impressive results have been reported in the treatment of patients suffering from ruptured aneurysm but the authors considered that selection of cases for admission and for operation might have given an over-optimistic impression of the impact of current methods of management. Their paper explored the ways in which data concerning a given patient population could be selected and presented in order to give different impressions of the efficacy of treatment. They contrasted in particular the operative mortality and the total management mortality. Their study was based on the outcome of 200 consecutive patients who suffered from ruptured aneurysm, and who had been drawn from 290 patients with a subarachnoid haemorrhage who had been investigated in a five and half year period. All patients under the age of 65 years with a subarachnoid haemorrhage had been transferred to their unit immediately after the patient was referred, regardless of the clinical condition. These represented $80-90 \%$ of the number of patients expected in their catchment area in that period. Seventeen per cent of the patients with an aneurysm had reached the neurosurgical unit within 72 hours of the last haemorrhage and 45 were in clinical grades 4 or 5 . Sixty-five per cent of patients with an aneurysm underwent operation, which in $70 \%$ was carried out after the tenth day. Analysis of the data had produced four main conclusions: (1) As compared with the natural history without treatment, there had been improvement of about $15 \%$ in the one year survival of patients with a ruptured aneurysm: (2) Although the overall management mortality was $35 \%$ at 1 year, delay in transferring cases or a more selective admissions policy would have reduced this by leading to the exclusion of a number of patients with a poor prognosis. The likely effect of various commonly applied policies for admissions on this patient population was estimated They could have led to an overall management mortality of as low as $16 \%$ at 1 year and a total surgical mortality of as low as $6 \%$ at 30 days: (3) The results of surgery could be presented in a number of ways, yielding operative mortalities ranging from $13.2 \%$ at 1 year (all cases who underwent surgery regardless of grade or the interval from the bleed) to $3.7 \%$ at 30 days ( 54 patients grades 1-3 at the time of surgery, operated on after the second week): (4) Examination of the causes of death both in patients who underwent surgery and those who did not, suggested that the methods of treatment currently available offer only limited prospects of further reduction of mortality. Improvements in the technical aspects of surgery appeared to offer the most hope for better results but a policy of much earlier surgery than had been applied would probably have had little impact on the overall outcome. This was because very few patients who died from rebleeding had been considered fit for operation.

FINAL OUTCOME AFTER ANEURYSM SURGERY PERFORMED IN THE ACUTE STAGE

Lennart Brandt, .Bengt Ljunggren, Hans Saveland (Sweden)

Many neurosurgeons are uncertain about whether a patient with a ruptured intracranial aneurysm is best treated by an operation either in the first 2-3 days after bleeding or several days later. The authors reported their experience with an early operation (within 72 hours) as a contribution to this debate. They had operated upon a consecutive series of 160 patients who were in Hunt and Hess neurological Grade I to III. One hundred and eighteen patients had made a good recovery but in 42 patients $(26 \%)$, the outcome had been unfavourable. The most common reason for this was delayed ischaemic cerebral dysfunction followed by a permanent deficit. This had occurred in 18 patients; $43 \%$ of all unfavourable results and $11 \%$ of the total series. An unfavourable outcome was due to surgical trauma in 11 patients $(26 \%$ of all unfavourable results; $7 \%$ of the total series) and to the initial bleed in seven patients ( $17 \%$ of all unfavourable results; $4 \%$ of the total series). The incidence of an unfavourable outcome was not related to the time of operation (day 1,2 or 3) but did correlate with a history of hypertension before rupture. Permanent ischaemic deficits occurred in $2 \%$ of 46 Grade I patients; in $13 \%$ of 78 Grade II patients; and in $19 \%$ of 36 Grade III patients. Intravenous administration of the calcium blocking agent, nimodipine, for at least 7 days after operation appeared to reduce the incidence of ischaemic deficits. The 
authors believed that it was principally the patient's condition in the early stage that determined the outcome. Early surgery with evacuation of blood-contaminated CSF and of clots, rather than increasing the risk of late cerebral dysfunction, might have reduced the frequency of this complication and nimodipine might further reduce its occurrence.

MULTIPLE: CEREBRAL ANEURYSMS-THE: LONGTERM OUTCOME AND IMPLICATIONS FOR TREATMENT

PJ Crawford, WS Almaain, SL Berga, JA Jane, AE Richardson, HR Winn (London) The dilemma in a patient who has suffered a subarachnoid haemorrhage and who is discovered to have multiple aneurysms is whether or not the unruptured lesion should be treated. In contrast to previous studies, which had largely concentrated on the short-term prognosis, this paper considered the long-term outcome of such patients and its implications for their management. As part of a controlled trial 182 patients were treated either by bed rest for 6 weeks or by craniotomy directed at the ruptured aneurysm. The incidence of late re-bleeding had been studied in 70 patients treated conservatively and 36 treated by operation who were followed for between 6 months and 21 years (mean 8 years). Thirty per cent of the conservatively treated patients and $27 \%$ of those operated upon had re-bled more than 6 months after the initial event. An extensive analysis showed that the incidence of late rebleeding in patients with a multiple aneurysm who had been treated conservatively was approximately $3 \%$ per year. This was therefore equal to that observed in patients with a single aneurysm treated similarly. Moreover, late haemorrhage in a patient with multiple aneurysms treated conservatively seemed always to occur from the original site of bleeding. The surgically treated patients had a rate of late haemorrhage due to the rupture of a previously intact aneurysm of approximately $1 \%$ per year. This was considered similar to that observed in patients with a single unruptured or incidental aneurysm. In both conservatively and operatively treated patients, an elevated blood pressure on admission had correlated with an increased risk of late re-bleeding. The results of this study provided a guide to decisions about the treatment of patients with multiple cerebral aneurysm. The authors concluded that the same critiera used to guide the management of a patient with a single aneurysm should be applied to determine if a patient with multiple aneurysms should be treated by an operation; moreover when performed, there might be no benefit in extending operation to aneurysms other than the source of the bleed if this in any way increased the risks of the procedure.

HPILEPSY AFTER (RANIOTOMY FOR INTRAC: RANIAL ANEURYSM

SA O'Laire, I Sbeith (London)

A consecutive series of 67 survivors of aneurysm surgery was analysed to determine the incidence of epilepsy six months to two and half years following operation. All patients had been given prophylactic anti-convulsant (300 $\mathrm{mg}$ phenytoin daily) with the intention of continuing treatment for one year, beginning 24 hours prior to surgery. Anticonvulsant levels were not monitored routinely. Thirteen patients ceased taking the phenytoin before the first out-patient review (2-3 months after surgery). All the patients were operated upon by one surgeon, who used standard microsurgical techniques. A slack brain was obtained by drainage of CSF by opening the basal cisterns; a preliminary ventricular tap was necessary in eight patients. Thirteen patients had multiple aneurysms and the distribution of aneurysms operated upon was: anterior communicating 23; posterior communicating 21; carotid 16; middle cerebral 13; anterior cerebral 4; basilar 3; posterior cerebral 1. Since operation two patients had each experienced a single grand mal fit; both had a history of previous epilepsy: one patient with an anterior communicating aneurysm had suffered febrile convulsions in childhood. and had a fit 24 hours after operation; one patient with a middle cerebral aneurysm had epilepsy following previous ECT, and suffered a fit 2 months after operation, whilst taking the prescribed anticonvulsant. The authors concluded that their findings were concordant with other recent reports that repair of an intracranial aneurysm by microsurgical technique was associated with a low incidence of post-operative epilepsy. They doubted the need for routine treatment with anticonvulsants and suggested a review of the regulations governing driving following craniotomy for intracranial aneurysm.

THE ROLE OF HISTOPATHOLOGY IN THE MANAGEMENT OF PITUITARY ADENOMAS

P Buxton, RV Jeffreys (Liverpool)

The modern management of pituitary adenoma involves endocrinologists, neuroradiologists, neurosurgeons and neuropathologists. There is an obvious interdependence between these groups although each group may also have a specific role. This presentation was concerned with the histopathological findings of 127 pituitary adenomas, each removed surgically. Patients were investigated endocrinologically before operation and placed in sub-groups as follows: acromegaly 41; anterior hypopituitarism; 40; normal pituitary function 31 ; prolactinoma 8 ; cushings 7 . Histological assessment was by conventional sections stained by haematoxylin and eosin and Slidders orange-fuchsin-green, by electron microscopy and by immunological staining for each of the six anterior pituitary hormones. Each of the 41 acromegalic patients had a raised serum growth hormone but on immuno-staining only $19 \%$ adenomas exhibited pure growth hormone $(\mathrm{GH})$, $31 \%$ showed a mixture of $\mathrm{GH}$ and prolactin (PRL) and 12\% PRL only, granules were seen under EM in $90 \%$ of tumours. Of the 40 adenomas from patients with hypopituitarism, $25 \%$ stained for PRL $\ddagger$ only, and $6 \%$ for luteinizing hormone $(\mathrm{LH})$ is and $6 \%$ for GH. Thirty-three percent $\vec{N}$ showed granules on EM. Of the 31 func- $\omega$ tionless adenomas $7 \%$ stained for PRL 음 only and $3 \%$ for a variety of hormone only $7 \%$ showed granules on EM. Each the patients with a presumed prolactinom had a raised serum PRL and each tumo showed granules on EM, but only $57 \overrightarrow{\mathbb{E}}$ stained for PRL. Similarly each of th seven patients with Cushing's Syndrome had a raised ACTH in the serum and granules on EM but only $57 \%$ of tumours stained for ACTH. As a result of the histopathological findings, nine cases of anterior hypopituitarism and two with a $\mathbb{D}$ functionless tumour were reclassified as a prolactinoma making 17 in all, compared to the pre-operative figure of eight. Two patients with anterior hypopituitarism had a tumour that appeared to be producing growth hormone. In conclusion it was felt that a combination of the histopathological techniques added greatly to the overall understanding of pituitary adenomas as well as contributing to the management of individual patients.

FERTILITY AFTER OPERATION FOR HYPER- 을 PROLACTINAEMIA

G Teasdale, A Richards, R Bullock, JA Thomson (Glasgow)

The restoration of fertility and the avoi- $N$ dance of complications in the event of $N$ pregnancy are major aims in the management of hyperprolactinaemic patients. To $\omega$ determine how often these were achieved $\underset{<}{\sigma}$ 
Table

\begin{tabular}{|c|c|c|c|c|c|}
\hline \multirow[b]{2}{*}{$\begin{array}{l}\text { Adenoma } \\
\text { Micro } \\
\text { Macro } \\
\text { None }\end{array}$} & \multirow[b]{2}{*}{$\begin{array}{r}N o \\
30 \\
7 \\
3\end{array}$} & \multicolumn{2}{|c|}{ Operation alone } & \multicolumn{2}{|c|}{ Operation + bromocriptine } \\
\hline & & $\begin{array}{l}\text { Pregnant } \\
18 \\
1 \\
2\end{array}$ & $\begin{array}{l}\text { Ovulation } \\
6 \\
1 \\
-\end{array}$ & $\begin{array}{l}\text { Pregnant } \\
4 \\
4 \\
1\end{array}$ & $\begin{array}{l}\text { Ovulation } \\
2 \\
-\end{array}$ \\
\hline
\end{tabular}

after operation the authors reviewed a series in whom the diagnosis of a prolactinoma was made on biochemical criteria and who had undergone transsphenoidal exploration. The patients had been evaluated by standard tests of anterior pituitary reserve and by determining the effects of TRH and metoclopramide on serum prolactin concentration.' If the prolactin increments were either abolished or markedly impaired $(<100 \%)$, the patient was offered operation, irrespective of the results of tomography or CT scan. In patients who had not conceived by the time of review, a late cycle rise in progesterone was taken to indicate ovulation. Seventy patients with amenorrhoea, galactorrhoea or infertility were explored, and a tumour was found in 63 . After operation $70 \%$ of the latter had a normal serum PRL $(<360$ $\mathrm{mu} / \mathrm{l})$. At review, 3-48 months postoperation, 40 patients declared that they had desired pregnancy, of these 30 had conceived. The table relates operative findings, post-operative treatment and fertility status.

Of the 42 pregnancies, only four miscarried and there were no major congenital malformations. None of the patients had symptoms that could be related to pituitary expansion during pregnancy; none received bromocriptine. Only three patients had serum PRL concentration above the normal for the phase of gestation. Of 10 patients who had not become pregnant, the post-operative serum PRL was normal in seven and in none was an endocrine deficiency responsible for the failure to conceive. The authors concluded that a transsphenoidal operation had enabled the majority of patients to achieve fertility and a trouble-free pregnancy. They also drew attention to the potential difficulties of operation for a micro prolactinoma, and stressed the need for further comparison with the short- and long-term results of medical treatment.

\section{Reference}

' Cowden EA, Thomson JA, Doyle D, Ratcliffe JG, Macpherson P, Teasdale GM. Test of prolactin secretion in diagnosis of prolactinomas. Lancet 1979;1:1155-8.
RECURRENCE OF MENINGIOMAS FOLLOWING SURGERY

SM Marks, A Kyriacou, RH Lye (Manchester)

The authors reviewed a consecutive series of 53 patients who underwent surgical removal of a meningioma during the period 1970-80. Their aim was to determine if there were features that predicted recurrence of the tumour. Ten of the patients were male and 43 were female. The average age was 52.9 years (range 26-87 years). Surgical removal was graded as: Type I-total macroscopic removal of tumour including its dural attachment and involved bone ( 21 patients); Type II-total removal and cautery of the dural attachment (11 patients); Type III-total removal with exploration of an involved venous sinus (6 patients); Type IVsubtotal removal (15 patients). Twelve patients received radiotherapy postoperatively; five after Type I, II or III procedures and seven after subtotal removal. The radiation dose was $3750-4250$ centigray. After surgery, patients were reviewed for an average of 5.3 years. There were nine deaths during this period, seven of which occurred in the first year; two patients died following Types I, II or III removals and seven patients following subtotal removals. The authors distinguished between recurrence (following Types I-III removal) and regrowth (after Type V removal). Recurrence rates were $9.5 \%$ (Type I) and $18.4 \%$ (Type II). There were no recurrences after a Type III operation. The average time between operation and detection of a recurrence was 2.6 years (range 1.6-3.8 years). Recurrence was suspected clinically in three patients and then confirmed radiologically; only in one instance did the CT scan identify an unsuspected recurrence. Regrowth following Type IV removal occurred in 10 patients $(66.6 \%)$. Although there was not a correlation between recurrence/regrowth and the age, sex or histological subtypes, tumours containing mitoses and areas of focal necrosis, which are indicators of active growth, had a tendency to recur or to regrow. The authors concluded that the factors of greatest significance in determining recurrence were the thoroughness of surgical removal and the absence of features of active growth. Their suggestion that a patient should undergo routine CT scanning at six months intervals during the first four years after removal of a meningioma provoked considerable controversy about the value of early detection of recurrence whilst a patient did not have symptoms.

EXTRADURAL PRESENTATION OF MENINGIOMATA

DA Campbell, JB Miles (Liverpool)

It is unusual for a meningioma to present extracranially. The authors had encountered three patients with a cranial meningioma that had presented with the symptoms of nasal obstruction; of 40 previous reports of a meningioma presenting extracranially (other than in the orbit) only one had presented with nasal obstruction. In the three patients that formed the basis of the authors' report, the diagnosis was made when specimens were examined histologically and the presence of the intracranial extension of the tumour had been appreciated only after the initial nasal operation. The difficulty of histological diagnosis was emphasised: a meningioma can mimic other tumours, so that a pathologist who was not experienced in neuropathology, might fail to recognise the diagnosis. The findings at operation in the three patients had suggested that the tumours had arisen from an extradural collection of arachnoid cells but a definite conclusion could not be drawn. The examples described had shown extensive involvement of adjacent structures and this had precluded total resection. Nevertheless the authors concluded that wide excision was important and that this could lead to a relatively good quality of survival.

DEVELOPMENTS IN ACOUSTIC NEUROMA SURGERY

L Symon, E Sayin, M Nitta (London)

The authors had reviewed 312 patients who had been operated on for removal of an acoustic neuroma. The survey covered 20 years and its aims were to review advances in techniques of treatment and changes in outcome. The total mortality was $5.7 \%$. In 189 patients treated before microsurgical techniques were used the mortality had been $7.9 \%$; subsequently the mortality in 123 patients was $2.4 \%$. The adoption of the operating microscope had been accompanied by a change from operation in a seated position to operation in the horizontal, park-bench position; other improvements in anaesthetic technique were noted. There was also a shift over the 
years to the treatment of more patients with a small tumour. The tumour had been completely removed in 273 patients but in 39 of the early cases only an intracapsular removal was performed. Three recurrences had occurred in patients treated before the operating microscope was used; subsequently there had been only one. There had also been an improvement in the rate of preservation of cranial nerve functions. Overall, the facial nerve was preserved anatomically in $46 \%$ of the 312 patients, the frequency of this achievement was related to the size of the tumour. Before the microscope was available the nerve was preserved in $39 \%$ of patients with a large tumour ( $>3 \mathrm{~cm}$.) and in $41 \%$ of these subsequently; in patients with a medium sized tumour $(1.5-3 \mathrm{~cm})$ preservation rates were $44 \%$ and $83 \%$ respectively; and in those with a small tumour $(<1.5 \mathrm{~cm}) 25 \%$ and $100 \%$. Facial nerve function had been assessed by a standard grading system in 37 of 52 patients in whom it had been preserved anatomically. Function was perfect or near perfect in eight of 12 patients who had a small tumour, in each of the five with a medium sized tumour and in seven of 20 with a large tumour. Facial movement could improve considerably for up to 2 years after operation. An attempt had also been made to preserve hearing in 50 patients. This was successful in seven of 10 with a small tumour, of whom five had functional hearing. This was found in one of four patients with a medium sized tumour and in one patient with a large but cystic tumour. The authors concluded that radiological, anaesthetic, and operative developments had improved the management of patients with an acoustic neuroma.

THE ROLE OF INTRAVENOUS DIGITAL SUBTRACTION ANGIOGRAPHY IN THE POST OPERATIVE ASSESSMENT OF INTRACRANIAL ANEURYSM OBLITERATION AFTER CLIPPING CEL Freer, RH Lye (Manchester)

The authors presented their early experience using intravenous digital subtraction angiography (DIVA) in the post operative assessment of the adequacy of intracranial aneurysm clipping. Pre-operative angiography revealed 10 aneurysms in nine patients. Clips were applied to nine aneurysms; a second aneurysm in one of the patients could not be found at operation. The clip position was considered satisfactory in all nine instances. Post-operative DIVA studies were attempted within the first week of surgery in eight patients. The procedure had to be abandoned in three patients because of technical difficulties such as lack of patient co-operation or difficulty in finding a suitable site for intravenous injection. Follow-up studies in these three, some weeks later, were performed without difficulty. In all postoperative DIVA studies, the clip was observed to have obliterated the fundus and neck of the aneurysm. It was possible to subtract the clip from the image to obtain better definition of the vessels in the vicinity of the aneurysm. Side effects were mild. The use of ionic contrast agents sometimes caused patient movement because of an intense sensation of heat, but this was avoided by using Iopamidol. Swallowing also produced movement artefact and re-masking was then essential. The authors discussed the advantages of DIVA studies and in particular, emphasised their safety in patients whose cerebral circulation might be precarious and in whom postoperative conventional angiography might be dangerous. They felt that digital subtraction angiography would eventually have a significant role in the management of patients with an intracranial aneurysm.

INTRAVENOUS DIGITAL SUBTRACTION ANGIOGRAPHY IN THE ASSESSMENT OF PATIENTS UNDERGOING CAROTID ENDARTERECTOMY

AH Kaye, JR Little, M Modic (Cleveland, Ohio)

The aim of this study was to evaluate the accuracy of pre-operative intravenous digital subtraction angiography (IDSA) in predicting the lesion found at operation for carotid artery disease. Particular attention was paid to signs of severe carotid stenosis or deep ulceration because these were considered to be the major radiological indications for carotid endarterectomy. Fiftythree patients who had 57 carotid operations were reviewed. Four studies that had been performed earlier in the series were considered technically unsatisfactory and the analysis was therefore based upon data from 53 studies. A severe stenosis of the carotid artery (more than $75 \%$ stenosis) had been found in 43 operations and in each case had been predicted correctly by the IV DSA. By contrast, although 30 of these arteries showed gross ulceration at operation, in only seven had this been shown by IV DSA. Conventional carotid angiography also under-estimated the frequency of ulceration in patients with severe stenosis and was positive in only nine of 26 patients who had been studied. Angiography did, however, predict ulceration in two patients more than did IV DSA. In nine carotid arteries the operation disclosed a combination of a moderate stenosis (50$74 \%$ ) along with deep ulceration. All of these ulcers had been predicted correctly by IV DSA. Carotid angiography was also correct in the five patients who had been studied. The authors reported more than 10000 IV DSA studies had been performed at their institute, and none of their patients had suffered a significant complication. They concluded that IV DSA could correctly demonstrate a severe stenosis of the carotid artery but that it would fail to show ulceration in such patients. However, it was reliable in identifying ulceration in the presence of either a moderate degree of stenosis of the carotid artery or in the absence of stenosis. They suggested that IV DSA adequately showed both the extracranial and intracranial major arteries and was an adequate and sufficient imaging technique in patients undergoing carotid artery operation.

THE ROLE OF NUCLEAR MAGNETIC RESONANCE (NMR) IN THE ASSESSMENT OF INTRACRANIAL AND INTRA-ORBITAL DISEASE: BS Worthington, RH Shephard, JC Taylor, it J Firth, M Gyngell, RC Hawkes (London) ' $\omega$ The authors had pioneered cranial NMR 윽 scanning and performed studies in over 400 patients with a wide spectrum of neurological disorders and compared th results with $\mathrm{x}$-ray computer tomograp and conventional neuroradiological proc dures. Expanding lesions produced chara teristic displacements and deformity of the ventricular system. For more precise identification of pathology they studied the pattern of tissue density when different spin sequences were applied, each being weighted differently by proton density and the relaxation times $T_{1}$ and $T_{2}$. The absence $\overrightarrow{\bar{\sigma}}$ of bone artefact was a significant advantage over computer tomography in the posterior fossa. The exquisite discrimination between grey and white matter shown on $T_{1}$ weighted images allowed the normal time course of myelination in the infant brain to be followed and facilitated the diagnosis of demyelinating disorders. The choice of 3 . imaging plane was achieved by methods that restricted data collection to the desired region allowing the perspective of direct coronal and sagittal images. This facility 음 allowed precise volumetric assessment and $D$ was useful in accurate localisation, as for 을. example in high convexity tumours. In the study of the peri-sellar region, of the brain $\mathrm{N}$ stem and of lesions impingeing on the mid- N line ventricular system, the sagittal plane was extremely valuable. The assessment of $\omega$ congenital and acquired abnormalities at or $\sigma$ 
adjacent to the craniovertebral junction was simplified by the use of NMR imaging and this allowed the more invasive procedures to be avoided. As with CT, orbital examinations displayed the globes, optic nerve and extra-ocular muscles contrasted against the retrobulbar fat. The multiplanar facility was useful in establishing the relationship of a tumour to normal structures. It was concluded that, although further evaluation of NMR imaging was required, it was clear already that it rep- : resented an important addition to existing techniques.

TRIPLANAR NUCLEAR MAGNETIC RESONANCE IMAGING OF CNS LESIONS IN ABERDEEN

DT Hope, FW Smith (Aberdeen)

Since 1982 the authors had used nuclear magnetic resonance (NMR) to image the brain of patients suspected of intracranial disease. Their scanner had been developed locally and utilised an 800 Gauss $(0.08$ Tesla) resistive magnet with a resultant NMR frequency of 3.4 MegaHertz for the hydrogen proton. Images were made by the "spin warp" technique and could be inversion recovery or calculated $T_{1}$, or proton density images. The resulting pictures were displayed on a video display unit and stored on floppy disk or film. The imaging time was either 25 seconds for proton density or 4 minutes to calculate data for all three of the parameters. Sections of the head were obtained in the primary sagittal, coronal and axial sections; the section thickness was 12 millimetres. They had aimed initially to image a wide spectrum of neurological diseases and to compare the findings with other conventional methods. The images obtained had been remarkably free from artefact. NMR imaging was particularly helpful in distinguishing lesions of the cervical cord, of the cerebellum and of the brain stem. The NMR images had shown characteristic plaques in patients suffering from multiple sclerosis whose CT scan had not shown abnormalities. Bilateral subdural haematomata had been identified in patients whose CT scan was unhelpful. The authors emphasised that the advantages of NMR imaging included the elimination of radiation, so that repeated day-to-day studies could be obtained if so desired, and the lack of interference artefact from bone. They speculated about future developments in NMR studies by their technique.

THE ROLE OF NUCLEAR MAGNETIC RESONANCE IN THE DIAGNOSIS OF INTRACRANIAL ANGIOMAS AND GIANT ANEURYSMS

P Lees, BS Worthington, RH Shepard, JC
Taylor, M Gyngell, RC Hawkes (London) The value of nuclear magnetic resonance (NMR) in specific disorders needs to be established. The authors had found that intracranial angiomas were clearly visualised and diagnosed with certainty on fast saturation recovery images. These highlighted blood vessels without the use of contrast media. Moreover, on steady state free procession images the moving blood led to removal of the signal. NMR scanning allowed angiography to be avoided when the site and extent of the angioma precluded operation. In other patients it gave useful anatomical information and complemented angiography. Haemorrhage and infarction were reliably assessed. NMR scans proved particularly useful in the assessment of giant intracranial aneurysms. Most patients had presented with features of an intracranial mass lesion and many of the aneurysms contained organised lamellated thrombus with only a small eccentric lumen demonstrated on angiography. NMR scans showed such aneurysms to have a smooth rounded margin and the thrombus within was clearly distinguished from blood flowing through the patent lumen. By using different scan sequences it was possible to prove that the area of low signal was indeed fluid in rapid motion and so to confirm the diagnosis with certainty. The authors concluded that NMR studies were very useful in patients suspected of having either an intracranial angioma or a giant aneurysm.

INTRACRANIAL INVESTIGATION OF THE MANCHESTER MUMMY HEADS

PA Stanworth, E Tapp, AR David, RA Fawcitt, H Jarvis, K Wildsmith (Manchester)

The Manchester Museum contains many fine examples of mummified Egyptian remains. The author and his colleagues had studied a specific group using non-invasive techniques. Six isolated human mummy heads had been examined radiologically, endoscopically and histologically. External appearances varied greatly, from one which contained parts of the original mask to another which was partially devoid of scalp tissue. Fluid levels were seen radiologically and were of two types. One had a sharp edge and was later shown to be due to resin that had pooled within the skull vault. A second irregular edge was seen, which turned out to be due to the outline of a mass of putrified brain. Defects were also seen on radiographs, and endoscopically in the base of some of the skulls, these were thought to have been made by the embalmer when extracting the putrified contents of the skull. One skull was devoid of soft tissue contents and yet had no route of entry other than the foramen magnum; the head may have been disarticulated from the body early on in the embalming process. Fragments of the soft tissues had been examined histologically, autolysed brain and parasites which had entered the heads after death were readily identified. The most interesting discovery had been a hydatid cyst within the putrified mass of brain in one of the heads (22940). Although hydatid disease has been seen in other tissue notably lung, previously it had not been described within a mummy head.

\section{ADVERSE EFFECTS OF LAMINECTOMY}

G Findlay (Liverpool)

Spinal cord compression due to a malignant tumour is usually managed by urgent laminectomy and subsequent radiotherapy. Radiotherapy alone has been employed, but there have been fears that, unless preceded by decompression, it could lead to neurological deterioration. Because he had noted that little attention had been given to the adverse effects of laminectomy, the author had reviewed thoroughly the literature from 1960 . He had utilised data only from papers which accurately identified the patients' neurological states before and after treatment. This had yielded more than 1800 patients, analysis of whom showed that overall only about one third were able to walk, regardless of whether treatment was by surgery or urgent radiotherapy. More surprisingly, one quarter of the patients had sustained major neurological deterioration and this had occurred equally frequently after laminectomy and radiotherapy. The author had postulated that vertebral collapse and instability might be important factors in producing adverse results; he had therefore reviewed 80 patients who had undergone a laminectomy for malignant compression of the thoracic cord, 41 of whom had radiological evidence of vertebral collapse. The findings before operation did not differ in those with or without vertebral collapse, and the results in those without collapse were similar to those in his extensive review; one third were able to walk but one quarter were worse after laminectomy. By contrast, only $15 \%$ of patients with vertebral collapse had walked after laminectomy and $50 \%$ had deteriorated by at least one functional grade. Only $8 \%$ of patients with vertebral collapse who were unable to walk had shown any functional improvement after operation whereas this had 
occurred in $27 \%$ of those without vertebral collapse. None of the patients without collapse had developed spinal instability but instability developed after laminectomy in $22 \%$ of those with collapse. The author suggested that laminectomy should no longer be considered automatically to be the first choice in therapy for malignant compression of the spinal cord. He suggested that comparable results could be achieved by giving the patient steroids and by instituting radiotherapy urgently and that improved results might follow if instability could be avoided.

CT OF THE SPINE: COMPARISON WITH MYELOGRAPHY AND CORRELATION WITH OPERATIVE FINDINGS

NI Azzam (Jeddah, Saudi Arabia)

The author reported a personal experience of 25 patients who had complaints thought to be of spinal origin and who were investigated by conventional myelography and by CT scan. Nineteen were found to have an intervertebral disc lesion and six had a spinal tumour. Eighteen of the 25 patients underwent operation. The CT findings correlated with the operative appearances in every patient. By contrast, metrizamide myelography was falsely negative in four patients and was inconclusive in three others. CT and myelography agreed in all patients with an extradural tumour but in only $14(74 \%)$ of those with a disc protrusion. Three of the negative myelograms were at the L5/S1 level and one at the L4/5 space. The results of this study were consistent with previous reports in which myelography had failed to demonstrate $15 \%$ of disc herniations at the lumbo-sacral level and a $5 \%$ of those at the L4/5 level. Although relatively few patients had been studied, the author believed that the conclusions were clear. He reported that CT was superior to myelography in the diagnosis of lumbar intervertebral disc protrusion, especially at the L5/S1 level. He suggested that patients with a strong clinical suspicion of a lumbar disc lesion but whose myelography was negative, should have CT of the spine. Myelography might be more useful in patients suspected of recurrence after operation. Although both the CT and myelography showed abnormalities in patients who had a tumour in the spinal canal, the former provided much more information about its position.

EVALUATION OF A NEW EXTRADURAL PRESSURE MONITORING SYSTEM

M Powell, A Crockard (London)

Intracranial pressure monitoring is in regular use in the management of head injuries, spontaneous intracerebral haematomas and in the diagnosis and management of normal pressure hydrocephalus. Intraventricular pressure (IVP) measurements give the most accurate records, but have the disadvantage of a high infection rate limiting useful monitoring time to two or three days; they also may be difficult to insert in a swollen or displaced brain. There are doubts raised about the validity of subdural pressure monitoring and extradural pressure (EDP) monitoring offers an attractive alternative. The authors had evaluated a commercially available system (Meadox Instruments) which measured extradural pressure as a function of resistance to air pumped constantly through an easily inserted extradural sensor, controlled by micro-circuitry to maintain a stable baseline. Two groups of patients were studied; in the first EDP was compared with IVP measured using a Kontron 0-60 $\mathrm{mmHg}$ external transducer, and in the second the frequency response and wave formation were assessed. The frequency response was observed to be rapid and the system recorded faithfully intracranial pressure changes of the same order as an intraventricular catheter. Simultaneous measurements were made of EDP and IVP in patients with low and high ICP (less than $10 \mathrm{mmHg}$ ). The system had no discernible baseline drift and the zero offset was unchanged before and after the study, but there was a tendency to over-estimate at low pressure and under-estimate when IVP pressure exceeded $25 \mathrm{mmHg}$. The authors considered that, although Meadox system showed promise, improvements would be necessary before it became a reliable aid to patient management.

WOUND CLOSURE BY ABSORBABLE SUBCUTANEOUS CONTINUOUS SUTURE AND ADHESIVE STRIPS TO SKIN

BH Cummings (Bristol)

Wound closure by black silk stitches is hallowed by long neurosurgical use but occa- sionally such wounds break down and more often they discharge pieces of silk long after the rest of the wound has healed. The author reported his experience of closure by means of a continuous subcutaneous suture with absorbable material and closure of the skin with adhesive strips (Steristrips). He had used this technique to close 1013 incisions made during 803 operations; 474 were closed with Dexon and 539 with Vicryl. Wound healing was uneventful in $96.6 \%$ and the overall infection rate was $1.6 \%$. In all types of operation, complications were infrequent. After 228 supratentorial craniotomies there was erosion of pericranial silk in four cases $(1.7 \%)$; one of these became infected. Two other patients with complicated problems developed wound infection $(1.3 \%)$. All these complications occurred in adults; by contrast only children had problems after a posterior fossa wound. Of 41 posterior fossa incisions seven $(17 \%)$ were complicated by the development of a bulging collection of CSF which ultimately ruptured and required shunting and re-suture; three of these became infected. In four patients the dura had been closed with silk and in three a lyodura patch was applied. It appeared that the original wound had healed well but hap broken down under pressure from CSF Eighty-two operations were to insert shunt; one wound discharged silk sutures from the flushing device and the other was eroded by the flusher itself. Both became infected with staphylococcus epidermidis $(2 \cdot 4 \%)$. Nine of 207 laminectomy incisions $(4.2 \%)$ were complicated; in four there was dehiscence; in three a haematoma; and in two a CSF leak. Staphylococci were grown in five cases. Of 169 cervical fusions (338 incisions) only one neck incision developed a haematoma $(0.6 \%)$ whereas seven iliac wounds $(2 \%)$ developed a haematoma and two became infected. Four of 74 incisions performed in a miscellaneous variety of operations were unsatisfactory $(5.4 \%)$. The author concluded that wound complications usually began deep, with either a haematoma or a collection of CSF and that extremely meticulous closure, probably with non-absorbable material, was needed when a CSF collection was likely. He had not found a difference in results with Dexon or Vicryl. He recommended the technique as usually resulting in excellent wound healing. 